\title{
The crosstalk between breast carcinoma-associated fibroblasts and cancer cells promotes RhoA-dependent invasion via IGF-1 and PAI-1
}

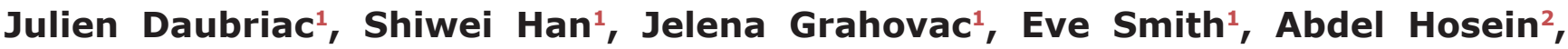 \\ Marguerite Buchanan'2, Mark Basik² and Yves Boucher ${ }^{1}$ \\ ${ }^{1}$ Edwin L. Steele Laboratory for Tumor Biology, Massachusetts General Hospital, Boston, Massachusetts, USA \\ ${ }^{2}$ Lady Davis Institute for Medical Research, Sir Mortimer B. Davis Jewish General Hospital, McGill University, Montreal, \\ Canada \\ Correspondence to: Yves Boucher, email: yves@steele.mgh.harvard.edu \\ Keywords: carcinoma-associated fibroblasts; RhoA/ROCK signaling; insulin-like growth factor-1; plasminogen activator inhibitor-1; \\ cancer cell scattering and invasion \\ Received: December 20, $2015 \quad$ Accepted: December 14, $2017 \quad$ Published: December 28, 2017 \\ Copyright: Daubriac et al. This is an open-access article distributed under the terms of the Creative Commons Attribution License \\ 3.0 (CC BY 3.0), which permits unrestricted use, distribution, and reproduction in any medium, provided the original author and \\ source are credited.
}

\section{ABSTRACT}

Carcinoma-associated fibroblasts (CAFs) can remodel the extracellular matrix to promote cancer cell invasion, but the paracrine signaling between CAFs and cancer cells that regulates tumor cell migration remains to be identified. To determine how the interaction between CAFs and cancer cells modulates the invasiveness of cancer cells, we developed a 3-dimensional co-culture model composed of breast cancer (BC) MDA-MB-231 cell spheroids embedded in a collagen gel with and without CAFs. We found that the crosstalk between CAFs and cancer cells promotes invasion by stimulating the scattering of MDA-MB-231 cells, which was dependent on RhoA/ ROCK/phospho MLC signaling in cancer cells but independent of RhoA in CAFs. The activation of RhOA/ROCK in cancer cells activates MLC and increases migration, while the genetic-down-regulation of RhoA and pharmacological inhibition of ROCK reduced cell scattering and invasion. Two distinct mechanisms induced the activation of the RhoA/ROCK pathway in MDA-MB-231 cells, the secretion of IGF-1 by CAFs and the upregulation of PAI-1 in cancer cells. In an orthotopic model of BC, IGF-1R inhibition decreased the incidence of lung metastasis, while Y27632-inhibition of ROCK enhanced the lung metastasis burden, which was associated with an increased recruitment of CAFs and expression of PAI-1. Thus the crosstalk between CAFs and BC cells increases the secretion of IGF-1 in CAFs and PAI-1 activity in cancer cells. Both IGF1 and PAI-1 activate RhoA/ROCK signaling in cancer cells, which increases cell scattering and invasion.

\section{INTRODUCTION}

In triple-negative breast cancer (TNBC) and other breast cancer subtypes, the presence of carcinomaassociated fibroblasts (CAFs) and collagen fibers correlates with high-grade malignancies and reduced survival [1]. CAFs have been shown to participate in tumor progression by enhancing invasion, metastasis, cancer cell survival and drug resistance $[2,3]$. This multiplicity of effects has to be put in perspective with the heterogeneity of the CAF population, as they have been shown to originate from resident fibroblasts, adipocytes, epithelial and endothelial cells as well as from bone marrow-derived mesenchymal and hematopoietic stem cells [3-5]. In comparison to normal stromal cells, CAFs display multiple molecular alterations that promote their proliferation and survival and modulate the secretion of cytokines and extracellular matrix (ECM) components $[6,7]$. In response to molecular signals from cancer cells (e.g. TGF- $\beta$, SDF-1) CAFs adopt an activated 
myofibroblast phenotype, characterized notably by an increased expression of $\alpha$-smooth muscle actin ( $\alpha$-SMA) and synthesis of collagen [8]. This desmoplastic reaction modulates the adhesion of cancer cells, leading to the remodeling of a permissive and supportive environment for tumor progression and dissemination [9-12].

Disseminating cancer cells undergo several molecular and cellular changes that modulate their intercellular and matrix adhesions as well as their mode of migration [13]. The loss of E-cadherin and the activation of epithelial-mesenchymal transition (EMT) are considered hallmarks of metastasis in several human cancers. In basal-like breast cancer the low expression of E-cadherin correlates with poor clinical outcome [14]. The loss of E-cadherin not only alters tissue homeostasis, allowing cancer cells to dissociate from the primary mass, but also promotes migration by mediating the activity of receptor tyrosine kinases [15]. CAFs have been shown to down-regulate the expression of E-cadherin and increase the migration velocity of breast cancer cells [16, 17].

Cancer cells display at least two distinct modes of single-cell migration, which are regulated by Rho family proteins (including RhoA, Rac1 and Cdc42) [18]. RhoA and its downstream effector ROCK mediate a proteolysis-independent amoeboid movement (rounded displacement) while Rac1 and Cdc42 drive the cells toward a mesenchymal type of migration (elongated displacement) [19-21]. The RhoA-dependent amoeboid movement is characterized by low-adhesion to matrix substrates and higher migration speeds, while Rac1dependent mesenchymal movement involves adhesion and proteolytical degradation of the ECM and is generally associated with lower migration speeds [19]. The amoeboid movement of cancer cells is a significant mode of migration in collagen gels in the absence of efficient pericellular proteolysis [22]. The disruption of the activity of RhoA or Rac1/Cdc42 is associated with malignancy $[20,23]$, and in invasive breast ductal carcinoma, the overexpression of either RhoA or Racl have been associated with worse prognosis [24]. In addition, ROCK1 and ROCK2 expression is increased in breast cancer specimens from patients with nodal metastasis $[25,26]$.

The architecture and composition of the ECM affect the mode of migration of cancer cells [22, 27, 28]. CAFs have been shown to remodel the ECM by creating tracks, which facilitate the collective migration of squamous carcinoma cells [29, 30]. The contractile forces that mediate matrix fiber alignment and stiffness are notably dependent on the expression of caveolin-1 and p190RhoGAP [11]. However, how the paracrine signaling between CAFs and cancer cells directly modulate the migration phenotype of cancer cells has not been studied in detail. To study how the interaction between CAFs (isolated from human invasive breast cancer including TNBC) and TNBC cells affects TNBC cell scattering and motility, intercellular contacts and cellular signaling, we developed a three-dimensional (3D) co-culture model of cancer cell spheroids and CAFs embedded in a collagen gel. Our results show that the crosstalk between cancer cells and CAFs promotes cellular scattering and invasion. The crosstalk between CAFs and cancer cells activates RhoA/ROCK signaling in cancer cells, which is dependent on the secretion of IGF-1 by CAFs and PAI-1 upregulation in cancer cells. We also investigated the significance of this crosstalk in a metastatic breast cancer model in mice.

\section{RESULTS}

\section{The crosstalk between CAFs and MDA-MB-231 cells promotes invasion and scattering}

The CAFs used for our experiments were isolated from a human invasive breast cancer of unknown subtype (CAF2; [31]), while the other CAF cell lines were isolated from human TNBC lesions [32] (see methods for additional information). To determine whether CAFs increase the invasion of BC cells, we incorporated MDAMB-231 breast cancer spheroids into a collagen gel with or without CAFs and measured cell scattering and the area of cancer cell invasion. Within 24 h CAF2 and 2 of 3 CAF TNBC cell lines induced a significant scattering of cancer cells, as determined by an increase in the number of single cells surrounding the spheroids (Figure 1A and 1B). On days 4 and 7, CAF2 and 3 of 3 TNBC CAF cell lines significantly increased the invasion of MDAMB-231 cells (Figure 1C and 1D). Cells that invaded the surrounding collagen gel had elongated or rounded shapes (Supplementary Figure 1A). There was no difference in cell aspect ratio (major axis / minor axis $=\sim 2$ ) or circularity (Supplementary Figure 1A) between spheroids cultured with or without CAFs.

\section{The crosstalk between $\mathrm{CAFs}$ and $\mathrm{BC}$ cells induce the activation of the RhoA/ROCK pathway in MDA-MB-231 cells}

Because the movement of rounded cells is linked to the activation of the RhoA/ROCK pathway, we determined whether CAFs could activate this pathway in MDA-MB-231 cells. We found that the co-culture of CAF2 and MDA-MB-231 cells increased the expression of RhoA-GTP in MDA-MB-231 cells (Figure 2A). We then assessed the effect of the ROCK inhibitor Y27632 on BC cell scattering and invasion. Y27632 did not affect the scattering and invasion of MDA-MB-231 cultured without CAFs, but significantly reduced the scattering (Figure 2B) and invasion induced by CAFs (Figure 2C, Supplementary Figure $1 \mathrm{~B}$ and $1 \mathrm{C}$ ), suggesting that CAFs promote the invasion of MDA-MB-231 cells via ROCK1/2. To confirm that CAFs promote cancer cell invasion by activating RhoA in MDA-MB-231 cells, we used shRNA interference to reduce the expression of RhoA in both 
cancer cells and CAFs (Supplementary Figure 1D and 1E). In RhoA-silenced cancer cell spheroids, CAFs did not increase invasion, confirming the role of RhoA-activation in promoting CAF-induced invasion (Figure 2D). RhoA silencing also reduced the invasion of MDA-MB-231 cells without CAFs, hence part of the invasion of MDAMB-231 cells is also dependent on the activity of RhoA. Because RhoA-dependent remodeling / contraction of the ECM by CAFs can promote cancer cell migration we also used shRNA constructs to knockdown the expression of RhoA in CAFs. The silencing of RhoA in CAFs reduced the expression of $\alpha$-SMA and MMP14 (Supplementary Figure 1E), but did not reduce the effects of CAFs on MDA-MB-231 cell invasion (Figure 2E). These findings suggest than in our 3D co-culture model, CAFs promote MDA-MB-231 invasion through secreted soluble factors rather than through a force-dependent remodeling of the ECM.

\section{TNBC cells increase the secretion of IGF-1 in CAFs}

In order to establish whether secreted factors could be responsible for CAF-promoted invasion, we measured by RT-qPCR array the transcription level of several genes related to EMT between CAFs and CAFs co-cultured with TNBC cells. In a transwell co-culture system (where CAFs and cancer cells were physically separated),

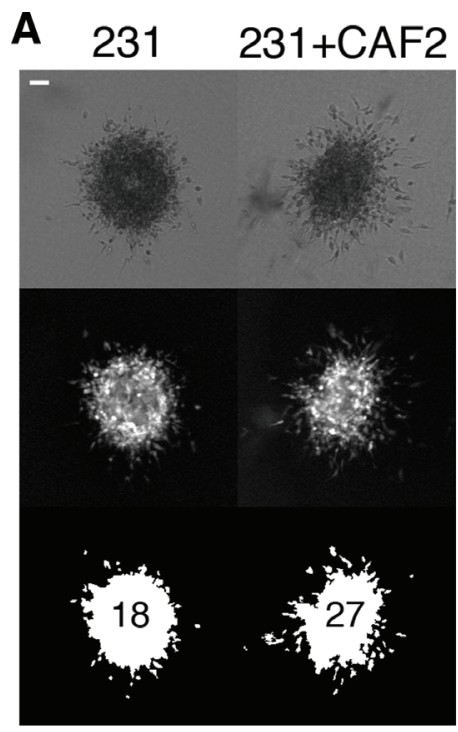

B
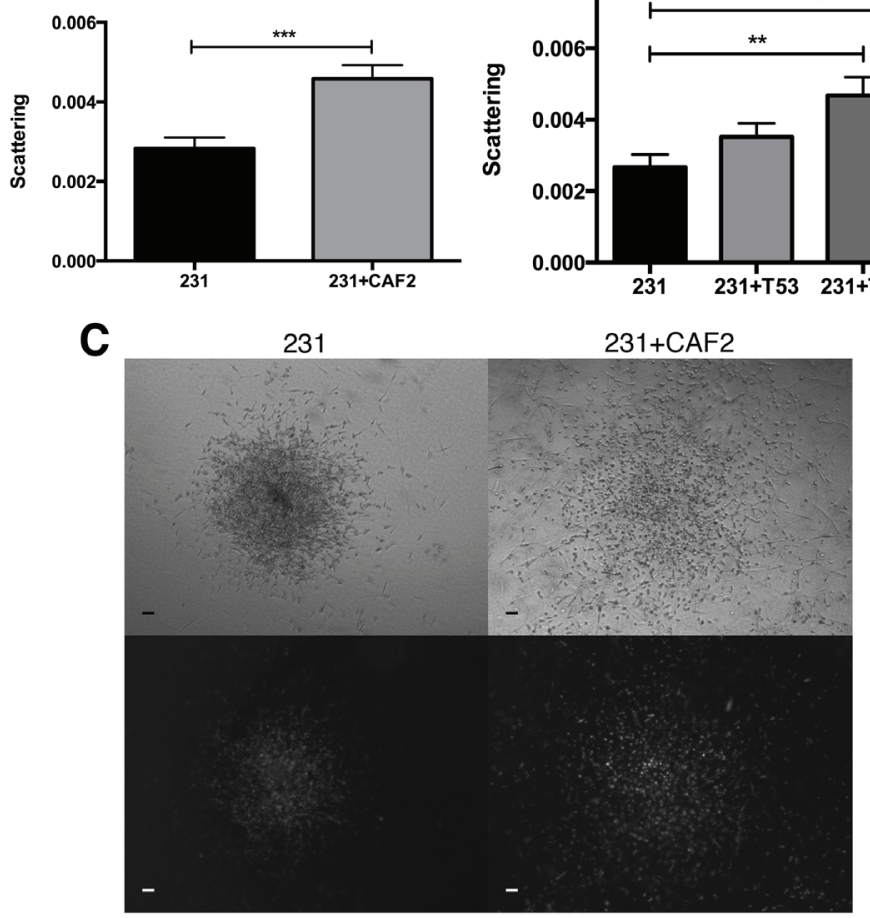

D
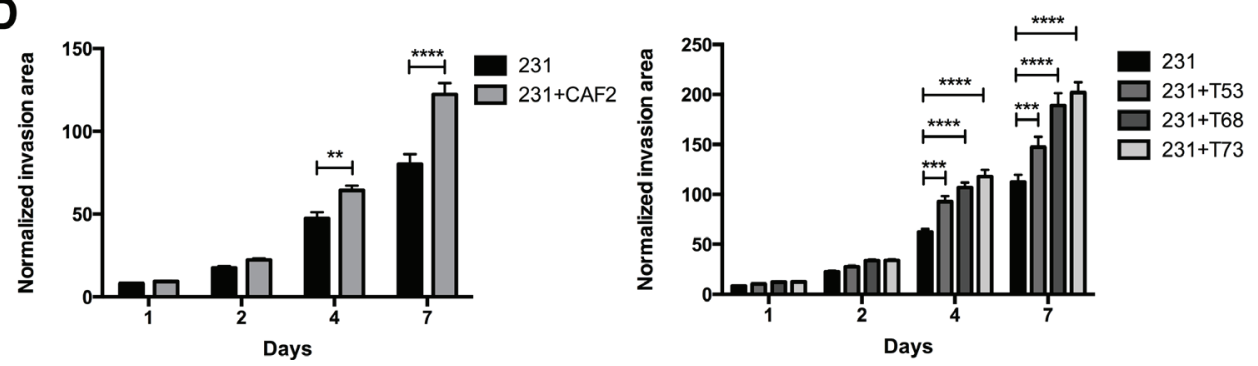

Figure 1: CAFs increase the scattering and invasion of MDA-MB-231 cancer cells. MDA-MB-231 spheroids cultured without or with CAF2 in a collagen gel over a 7-day period. (A-B) At day 1, scattering was evaluated by counting the number of GFP ${ }^{+}$ single MDA-MB-231 cells surrounding the spheroid. (C) Kinetic of GFP ${ }^{+}$MDA-MB-231 cells invasion with or without CAFs in collagen gels: The normalized invasion area was defined as the GFP ratio of the invasion area to the size of the spheroid at day 0. Brightfield (top) and fluorescent (bottom) images showing spheroids and the invasion of 231 cells at distance from center of spheroids. (D) On day 4 and 7 CAFs significantly increased the invasion of MDA-MB-231 cells. Data expressed as mean \pm SEM. ${ }^{*} p \leq 0.05,{ }^{* *} p \leq 0.01,{ }^{* * *} p \leq 0.001$, ${ }^{* * * * *} p \leq 0.0001$. Bar $100 \mu \mathrm{m}$. 
MDA-MB-231 cells increased the transcription of $I G F 1$ by 12 fold in CAFs (Figure 3A). In MDA-MB-231 cells alone or co-cultured with CAFs, IGF1 could not be detected $(\mathrm{Ct}>35)$ (Supplementary Figure 2A). The expression of $D P P 4$ and ITGB3 in CAFs were both increased by 2.5 fold, while the expression of $E G F, F G F 2, H G F$ or $T G F B 1$ was not affected by MDA-MB-231 cells (Supplementary Figure 2B). Next, we measured by ELISA the secretion of IGF-1 in the supernatant of CAFs alone or co-cultured with cancer cells. MDA-MB-231 cells significantly increased the secretion of IGF-1 in all CAFs tested, but did not affect the expression of IGF-1 in a normal fibroblast cell line (Figure 3B). The TNBC cell line MDA-MB-436 also increased the secretion of IGF-1 in CAF2 (Figure 3B).

\section{IGF-1 increases RhoA signaling and invasion in MDA-MB-231 cells}

We then tested the effect of recombinant IGF-1 on cancer cell invasion. IGF-1 [50 nM] significantly increased the invasion of MDA-MB-231 cells (Figure 3C). Reciprocally, antibody blockade of IGF-1 significantly reduced the $\mathrm{CAF}$-enhanced scattering and invasion of MDA-MB-231 cells (Figure 3D and 3E). To determine whether IGF-1 promotes cancer cell invasion via the RhoA/ROCK pathway, we examined the effect of recombinant IGF-1 on RhoA-GTP expression. IGF-1 [50 $\mathrm{nM}$ ] activated RhoA within $15 \mathrm{~min}$ (Figure 3F) and the IGF-1 blocking antibody reduced CAF-induced RhoAGTP expression in MDA-MB-231 cells (Figure 3G). Finally, we tested the effect of recombinant IGF-1 on invasion in RhoA-silenced MDA-MB-231 cells. In comparison to mock-transfected cells, IGF-1 did not increase the invasion of RhoA-silenced cells (Figure 3C and $3 \mathrm{H})$. Hence, our results show that TNBC cells increase the CAF-secretion of IGF-1, which enhances RhoA/ ROCK-dependent invasion.

To assess the role of adherens junction proteins - in CAF-promoted scattering of tumor cells - we examined the expression of E-cadherin and phospho-p120 catenin, which regulates RhoA activity. In particular, the phosphorylation of Tyr228 increases the binding affinity of p120 catenin for RhoA, maintaining RhoA in an inactive (GDP) form [33]. Three of 3 CAFs and IGF-1 reduced
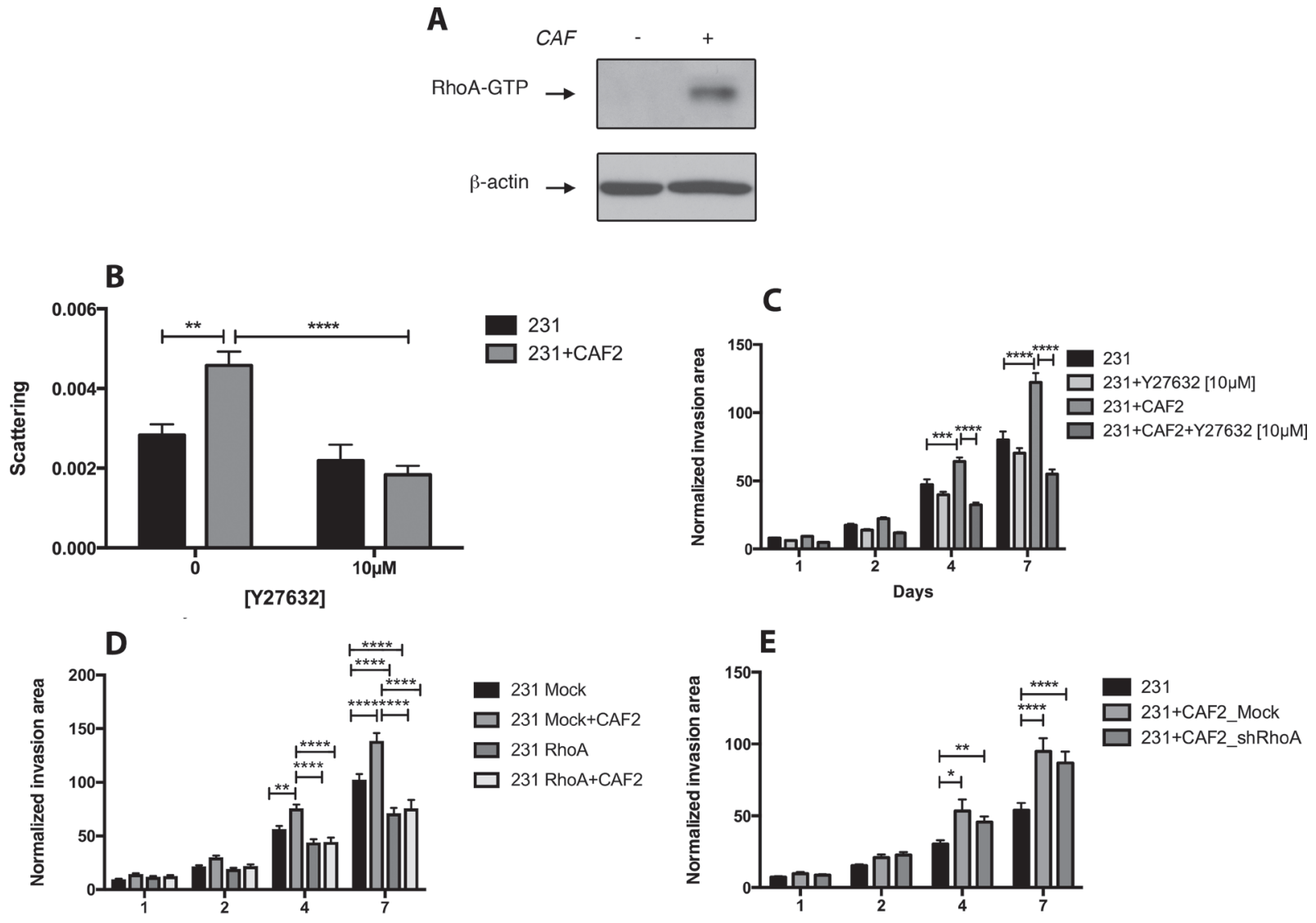

Figure 2: CAFs promote MDA-MB-231 invasion and scattering by activating RhoA/ROCK in cancer cells. (A) Effect of CAFs on RhoA-GTP expression in MDA-MB-231 cells: MDA-MB-231 spheroids were culture with or without CAF2 for $72 \mathrm{~h}$ and assayed for RhoA activation by RhoA-GTP pulldown assay. $\beta$-actin was used as a loading control. (B-C) Effect of Y27632 [10 $\mu \mathrm{M}]$ on the scattering and invasion of MDA-MB-231 cells cultured with or without CAF2 in a collagen gel. (D) Kinetic of RhoA-silenced MDAMB-231 cells invasion with or without CAFs in collagen gel. (E) Kinetic of GFP ${ }^{+}$MDA-MB-231 cells invasion with RhoA-silenced or mock-transfected CAFs in collagen gel. Data expressed as mean \pm SEM. ${ }^{*} p \leq 0.05,{ }^{* *} p \leq 0.01,{ }^{* * *} p \leq 0.001,{ }^{* * * *} p \leq 0.0001$. 
the expression of E-cadherin and the phosphorylation of p120 catenin although to different extents (Supplementary Figure 2C and 2D). Interestingly, the addition of Y-27632 to either co-cultures of CAF2 and MDA-MB-231 or IGF1-treated MDA-MB-231 cells increased the expression of E-cadherin and phospho-p120 catenin, which suggest that their downregulation is ROCK-dependent. However, immunofluorescence indicated that E-cadherin is mostly expressed in the cytoplasmic compartment in MDAMB-231 spheroids suggesting that E-cadherin might not have a significant effect on cell-cell adhesion or in hindering the scattering or invasion of MDA-MB-231 cells in presence of CAFs (Supplementary Figure 2E).

\section{CAFs increase PAI-1 expression in MDA- MB-231 cells independently of IGF-1}

To determine whether the crosstalk between cancer cells and CAFs modulate EMT, migration pathways in cancer cells we analyzed the expression of 84 EMTrelated genes with a PCR array. The co-culture of CAF2 and MDA-MB-231 cells enhanced the transcription of
SERPINE1 (PAI-1) (Figure 4A). CAF2 also increased the expression of KRT19 by 10 -fold and MMP3 by 6 -fold in MDA-MB-231 cells (Supplementary Figure 3A). At the protein level, we confirmed that all CAFs and also normal fibroblasts increased levels of the full-length form of PAI-1 in MDA-MB-231 cells (Figure 4B). However, IGF-1 did not increase the expression of PAI-1 in MDAMB-231 cells. Of note, MDA-MB-231 cells also express a lower molecular band of PAI-1 that could correspond to a truncated form of the protein.

Next, we tested the effect of geodin - a small molecule that inactivates PAI-1 - on cell scattering and invasion. At $\left[\begin{array}{ll}10 & \mu \mathrm{M}\end{array}\right]$, geodin had minimal effects on cell viability of CAFs and MDA-MB-231 cells (Supplementary Figure 3B-3C), but reduced the scattering and invasion of MDA-MB-231 cells, independently of the presence of CAFs (Figure 4C4D, Supplementary Figure 3E), indicating that the basal expression of PAI-1 is required for invasion. In comparison to Y27632 inhibition of ROCK and antibody blockade of IGF-1, geodin produced a greater inhibition of cell scattering (Geodin 80\%, Y27632 54\%, IGF-1 24\%).
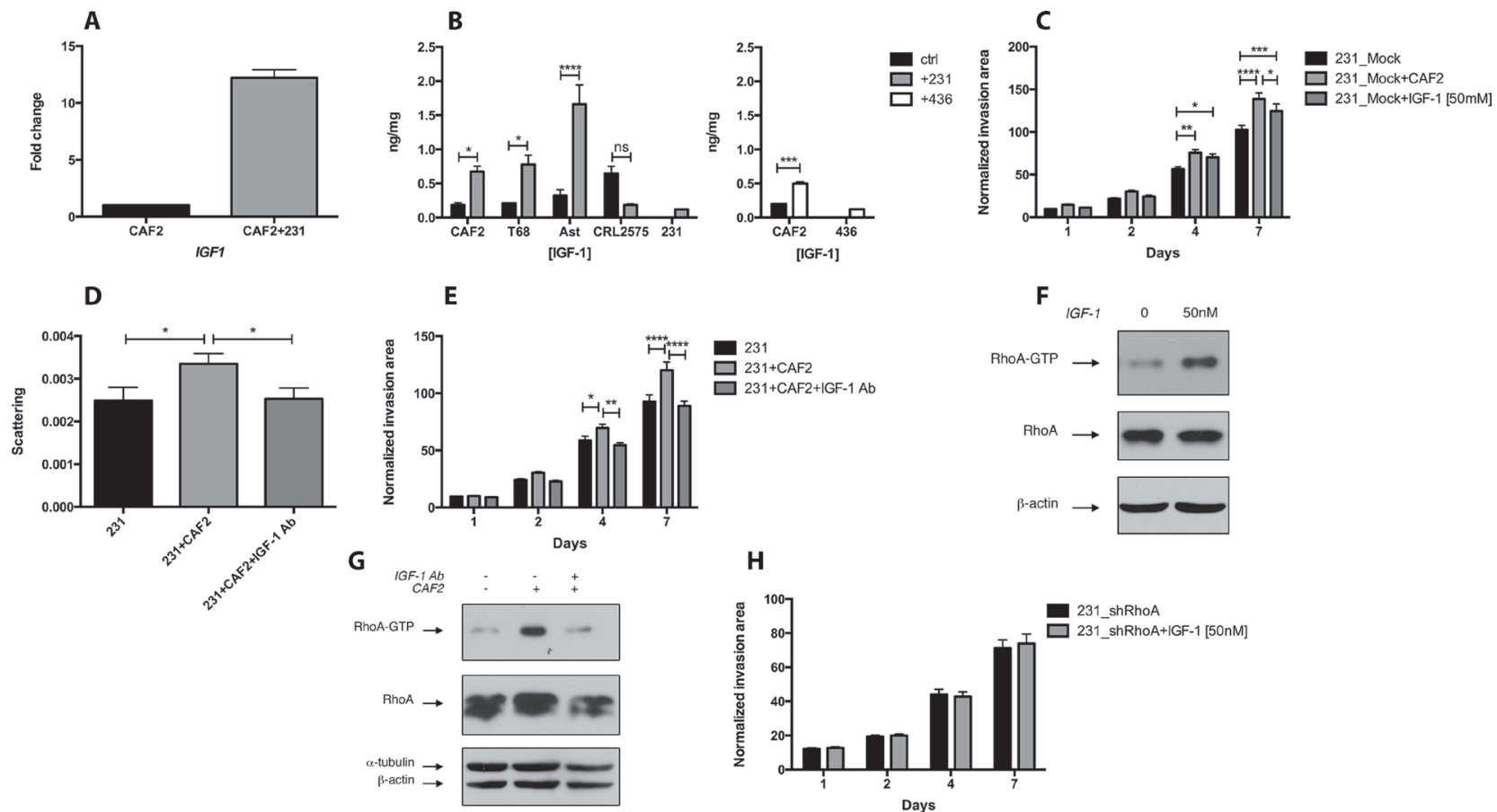

Figure 3: CAFs promote RhoA/ROCK-dependent invasion and scattering of MDA-MB-231 cells via IGF-1. (A) CAF2 were cultured with or without MDA-MB-231 cells for $72 \mathrm{~h}$ and assayed for IGF1 expression by RT-PCR. MDA-MB-231 cells increase IGF1 in CAF2 by 12 fold. (B) Similarly, several CAFs and CRL2575 cells were cultured with or without MDA-MB-231 or MDA-MB-436 cells for $72 \mathrm{~h}$ in serum-free condition, and supernatants were assayed for IGF-1 expression by ELISA. MDA-MB-231 increased IGF-1 secretion in all CAFs tested but not in CRL2575. MDA-MB-436 cells increased IGF-1 secretion in CAF2. (C) Effect of recombinant IGF-1 [50 mM] on collagen invasion of MDA-MB-231. (D-E) Effect of IGF-1 blocking antibody [20 $\mu \mathrm{g} / \mathrm{ml}]$ on scattering and invasion of MDAMB-231 cells cultured with or without CAF2 in collagen gel. (F) Effect of recombinant IGF-1 on RhoA-GTP expression in MDA-MB-231 cells: MDA-MB-231 spheroids were culture with or without $[50 \mathrm{nM}]$ recombinant IGF-1 for 15 min and assayed for RhoA activation by RhoA-GTP pulldown assay. (G) Effect of IGF-1 blocking antibody on RhoA-GTP expression in MDA-MB-231 cells: MDA-MB-231 spheroids were culture with or without CAF2 and treated with an IGF-1 blocking antibody for $72 \mathrm{~h}$ and assayed for RhoA activation. (H) Effect of recombinant IGF-1 on collagen invasion of RhoA-silenced MDA-MB-231 cells. Data expressed as mean \pm SEM. ${ }^{*} p \leq 0.05$, ${ }^{* *} p \leq 0.01,{ }^{* * *} p \leq 0.001,{ }^{* * * *} p \leq 0.0001$. 
We also tested the effect of geodin on the highly invasive murine breast cancer cell line 4T1, which does not express PAI-1. Geodin did not affect the invasion of 4T1 cells (Supplementary Figure 3F).

To determine whether RhoA activation was dependent on PAI-1 expression, we analyzed the effect of geodin $[10 \mu \mathrm{M}]$ on the expression of RhoA-GTP and phospho-MLC (Thr18/Ser19). Geodin decreased the expression of RhoA-GTP and phospho-MLC in MDAMB-231 cells cultured with or without CAFs, suggesting that RhoA-activation by PAI-1 is linked to the expression of PAI-1 (Figure 4E). Interestingly, PAI-1 expression increased in cells treated with Y27632, suggesting the existence of a regulatory loop between PAI-1 and ROCK (Figure 4B). Furthermore, the expression of PAI-1 was not affected by IGF-1, which indicates that CAFs activate RhoA in MDA-MB-231 cells via two distinct pathways, the secretion of IGF-1 by CAFs and the upregulation of PAI-1 in MDA-MB-231 cells.

\section{Targeting IGF-1R, but not ROCK, reduces the incidence of MDA-MB-231 lung metastasis without affecting tumor growth}

In order to determine if targeting different components of the IGF-1/RhoA/ROCK crosstalk between $\mathrm{BC}$ cells and CAFs would affect primary tumor growth and metastasis, we treated MDA-MB-231 xenografts with the IGF-1R inhibitor PQ401 [34], Y27632 or PQ401 combined with Y27632. After 18 days of treatment, we did not observe any effect of PQ401, Y27632 or PQ401 combined with Y27632 on primary tumor growth (Figure 5A), suggesting that the inhibition of ROCK or IGF-1 receptor did not affect cancer cell proliferation.

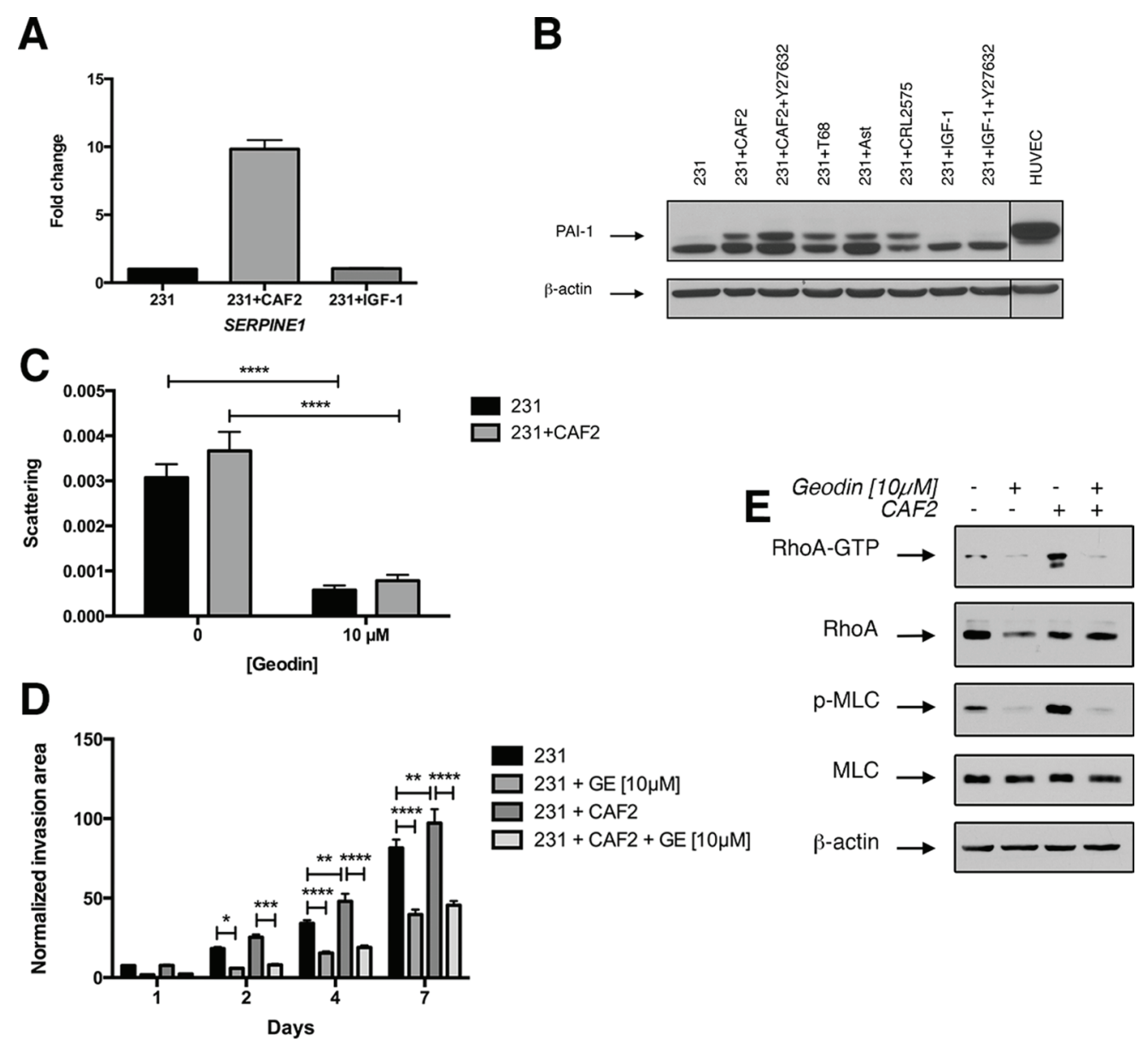

Figure 4: CAFs but not IGF-1 increase PAI-1 expression in cancer cells. (A) MDA-MB-231 spheroids were culture alone or in presence of CAFs or IGF-1 [50 nM] for $72 \mathrm{~h}$ and assayed for SERPINE1 (PAI1) expression by RT-PCR. (B) MDA-MB-231 cell spheroid were cultured alone or in presence of CAFs, IGF-1 [50 nM] and/or Y27632 [10 $\mu \mathrm{M}]$ for $72 \mathrm{~h}$ and immunoblotted for PAI-1 and $\beta$-actin as loading control. HUVECs were used as a positive control for PAI-1 expression. (C-D) Effect of PAI-1 inhibition on scattering and invasion of MDA-MB-231 cells in a collagen gel. MDA-MB-231 spheroids cultured with or without CAF2 in a collagen gel over a 7-day period in absence or in presence of $10 \mu \mathrm{M}$ of geodin. (E) Effect of PAI-1 inhibition on RhoA pathway activation in MDA-MB-231 cells: MDAMB-231 cells were cultured alone or in presence of CAF2 in absence or presence of $10 \mu \mathrm{M}$ of geodin and assayed for RhoA activation by RhoA-GTP pulldown assay and immunobloted for phospho-MLC (Thr18/Ser19). Data expressed as mean \pm SEM. ${ }^{*} p \leq 0.05,{ }^{* *} p \leq 0.01$, ${ }^{* * *} p \leq 0.001,{ }^{* * * *} p \leq 0.0001$. 
Five weeks after the resection of primary tumors, we analyzed the incidence of mice with metastasis and the metastasis burden in lung sections (fraction of lung tissue occupied by tumor). In the control group and mice treated with either Y27632 or Y27632 combined with PQ401, all mice showed evidence of macroscopic lung metastasis (Figure 5B). In contrast, in the PQ401-treated group the incidence of macroscopic lung metastasis was significantly less ( $p=0.0351$, Chi-squared test). Four of 12 mice had no macroscopic disease ( 3 mice had no lung metastasis and 1 mouse had microscopic disease) (Figure 5B). In comparison to the vehicle, PQ401-treated mice showed a $28 \%$ decrease in lung metastatic burden, the difference was not significant ( $p=0.30$, Two-way ANOVA), while Y27632 alone or combined with PQ401 significantly increased the metastatic burden $(p=0.0434$, Two-way ANOVA) (Figure 5C).

\section{Inhibition of IGF-1 signaling and ROCK increases the expression of E-cadherin and phospho-p120 catenin in MDA-MB-231 tumors}

To assess whether the inhibition of ROCK or IGF$1 \mathrm{R}$ was associated with change in adherens junctions as we observed in vitro, we analyzed the expression of E-cadherin and phospho-p120 catenin (Tyr228) by
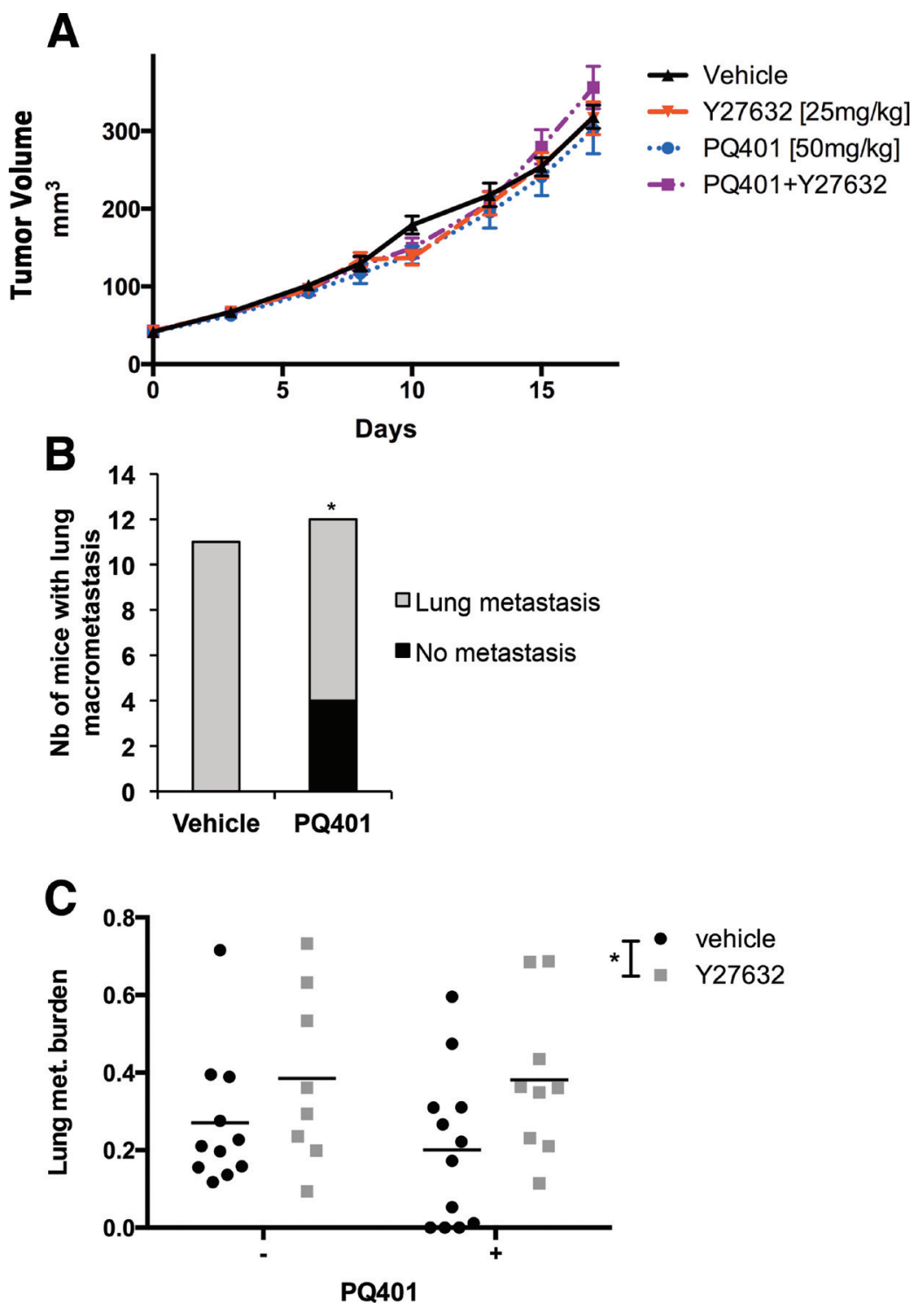

Figure 5: IGF-1R inhibition but not ROCK inhibition reduces the incidence of lung metastasis without affecting tumor growth in breast cancer xenografts. (A) MDA-MB-231 cells were implanted in the mammary fat pad of SCID mice and treated with PQ401, Y27632 or PQ401 combined with Y27632. IGF-1R and ROCK inhibition did not affect primary tumor growth. (B-C) Five weeks after primary tumor resection, lungs of MDA-MB-231 tumor bearing mice were collected and analyzed for lung metastasis. PQ401 decreased non-significantly the metastasis burden in lungs, but significantly decreased the incidence of lung metastasis. Y27632 alone or combined with PQ401 significantly increased the lung metastasis burden. 
immunoblotting tumor lysates. We found that Y27632 significantly increased the expression of E-cadherin $(p<0.0001)$ and phospho-p120 catenin $(p=0.0071)$ (Supplementary Figure 4). In PQ401-treated tumors, there was a trend for an increased expression of E-cadherin ( $p=$ 0.0546 ) while only 3 tumors showed high phospho-p 120 catenin expression. Moreover, when combined to PQ401, the effect of Y27632 on both E-cadherin, phospho-p120 catenin and PAI-1 expression was significantly diminished, indicating a possible interaction between the two drugs.

\section{Inhibition of ROCK increases the recruitment of CAFs and PAI-1 expression in MDA-MB-231 tumors}

First, to validate the effect of IGF-1R inhibition on RhoA signaling, we quantified the level of RhoAGTP expression in tumors treated with PQ401. We found that the PQ401-treated group showed a $24 \%$ decrease in RhoA-GTP level in comparison to the control group (ns) (Figure 6A). Then, to determine whether the level of PAI-1 was affected by the inhibition of ROCK in primary tumors, we analyzed its expression in tumor lysates. Similar to our in vitro findings, Y27632 significantly increased the expression of PAI-1, confirming the existence of a regulatory mechanism between ROCK and PAI-1 in vivo (Figure 6B and 6C). PQ401 induced a minor but significant increase in PAI-1 expression, while PQ401 combined with Y27632 did not affect PAI1 expression. To better understand the difference in lung metastasis incidence between Y27632 and PQ401, we quantified the $\alpha$-SMA expression in primary tumors by immunohistochemistry. In contrast to our in vitro data showing that RhoA down-regulation reduces $\alpha$-SMA expression in CAFs, Y27632 significantly increased the number of $\alpha$-SMA-positive cells, independently of the presence of PQ401, while PQ401 reduced the number of $\alpha$-SMA-positive cells but not significantly (Figure 6D and $6 \mathrm{E})$. Moreover, we found that the expression of the EMT marker phospho-ERM (Thr567/564/558) was increased by Y27632 but not by PQ401 (Figure 6F and 6G). Our results suggest that ROCK-inhibition in primary tumors promotes the recruitment of $\alpha$-SMA-positive CAFs and expression of PAI-1, which may increase the invasiveness of cancer cells.

\section{DISCUSSION}

Our findings show that in a 3D collagen gel the crosstalk between CAFs and BC cells promotes the
A
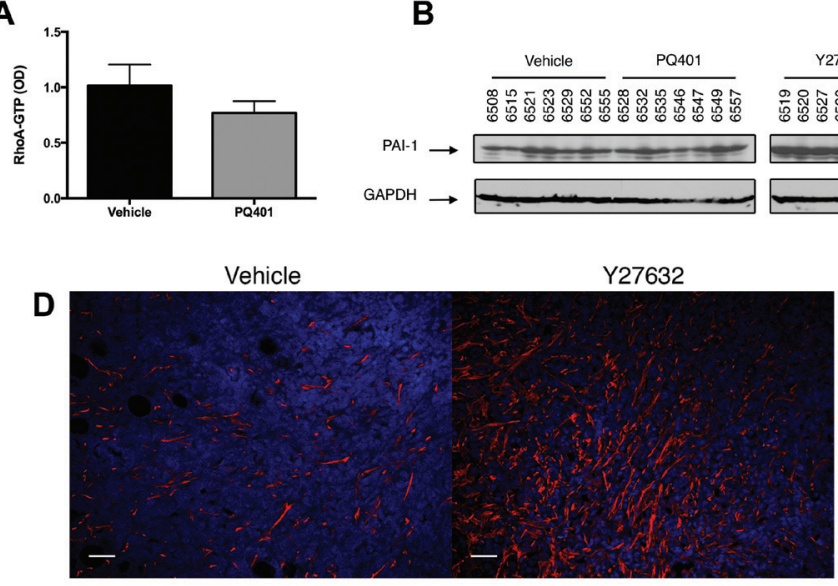

Vehicle

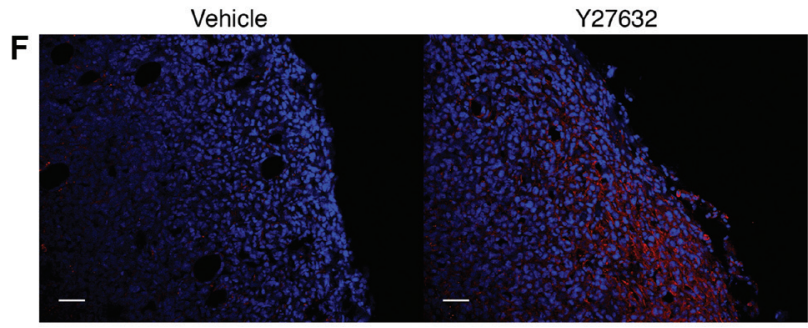

B

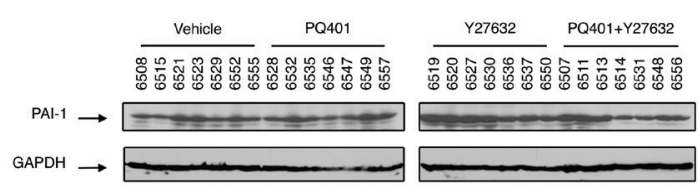

E
G

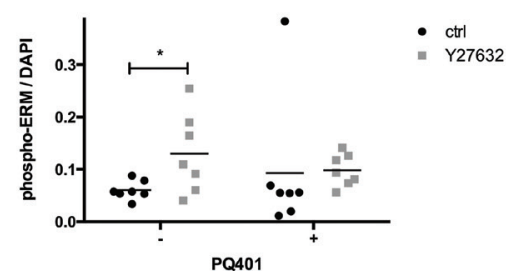

Figure 6: ROCK inhibition increases the recruitment of CAFs and PAI-1 expression in breast cancer xenografts. (A-C) After 18 days of treatment, tumors were harvested and assayed for RhoA activation by G-LISA colorimetric assay ( $n=6$ per group), and for PAI-1 expression by immunoblotting. (D, F) Primary tumor sections were immunostained for $\alpha$-SMA and phospho-ERM. (E, G) Y27632 or Y27632+PQ401 increases the number of $\alpha$-SMA-positive cells in MDA-MB-231 primary tumors while only Y27632 significantly increases phospho-ERM expression. Bar $50 \mu \mathrm{m}$ Data expressed as mean \pm SEM. ${ }^{*} p \leq 0.05,{ }^{* * * *} p \leq 0.0001$. 
invasion of MDA-MB-231 cells by stimulating RhoA GTPase, cell scattering and migration. In our model, this modulation of cancer cell invasiveness is not mediated by RhoA-dependent contractile forces of CAFs [11, 29, 30]. Instead the crosstalk between MDA-MB-231 cells and CAFs leads to increased migration, which depends on the activation of RhoA/ROCK/MLC signaling in cancer cells via two distinct mechanisms, the secretion of IGF-1 by CAFs and the upregulation of PAI-1 in cancer cells (Supplementary Figure 5).

IGF-1 signaling is known to induce EMT and promote invasion in $\mathrm{BC}$ cells [35]. The elevated expression of stromal IGF-1 and CXCL12 in primary TNBC lesions from patients has been linked with the selection of clones with high Src activity and bone metastasis formation [36]. Furthermore, the pharmacological - as shown in the present study - and genetic inhibition of IGF-1R reduces the incidence of lung metastasis [37]. Similar to our findings showing that $\mathrm{BC}$ cells increase the secretion of IGF-1 in CAFs, the tumor-conditioned media of TNBC cell lines - including MDA-MB-231 cells - stimulates the expression IGF-1 in human mesenchymal stem cells [36]. We show here that IGF-1 secretion by CAFs activates RhoA signaling in MDA-MB-231 cells, which promotes cell scattering and invasion. Interestingly, CAFs isolated from breast cancer lesions also secrete IGF binding proteins, which increases anoikis-resistance [38]. IGF-1R signaling also promotes the survival and apoptosis resistance [39]. Thus these findings suggest that the secretion of IGF-1 by CAFs supports the scattering, invasion and survival of BC cells during the initial steps of the metastatic cascade.

Independently of IGF-1, the crosstalk between CAFs and $\mathrm{BC}$ cells also increased the expression of PAI1 in MDA-MB-231 cells, while geodin-inhibition of PAI-1 reduced RhoA activity in cancer cells, cancer cell scattering and invasion. Furthermore, geodin also reduced the scattering and migration of unstimulated MDAMB-231 cells - without CAFs -, which highlights the importance of PAI-1 in regulating the migration of MDAMB-231 cells. PAI-1 can also promote the proliferation and survival of cancer cells [40], EMT [41], the migration and invasion of BC cells [42] and the amoeboid migration of colorectal cancer cells [43], as well as angiogenesis [44]. PAI-1 expression in cancer cells is regulated by TGF- $\beta[45,46]$. CAFs secrete TGF- $\beta$ to promote EMT in cancer cells [17]. Therefore, in parallel to IGF-1, TGF- $\beta$ could contribute to the RhoA-dependent enhancement of cancer cell invasiveness via PAI-1.

The RhoA/ROCK pathway has been described in normal cells as a regulator of PAI-1 expression [47]. In contrast, our results show that geodin-inhibition of PAI1 decreases RhoA activity in MDA-MB-231 cells and phospho-MLC (downstream target of RhoA) in HUVECs (Supplementary Figure 3G). Furthermore, we found that ROCK inhibition with Y27632, in parallel to the inhibition of its downstream target MLC (Supplementary Figure 6), increases both in vitro and in vivo the expression of PAI1 , suggesting the existence of a feedback loop between ROCK and PAI-1.

Although we identified a correlation between RhoA activation and tumor volume doubling time in vivo (Supplementary Figure 7), the Y27632 inhibition of ROCK did not affect primary tumor growth. Similar to our Y27632 findings, the knockout of either ROCK1 or ROCK2 in MDA-MB-231 cells did not affect tumor growth, whereas the intratumoral injection of anti-RhoA siRNA reduced tumor growth [48, 49]. Together, this suggests that the effect of RhoA on tumor cell proliferation could be independent of its downstream effectors ROCK1 and ROCK2. Another RhoA effector, mDia1, which works independently of ROCK, has been shown to contribute to cell cycle progression [50]. Contrary to our in vitro findings showing that the targeting of either RhoA or ROCK reduces CAF-enhanced invasion, the treatment of MDA-MB-231 xenografts with Y27632 increased lung metastasis. ROCK inhibition is known to facilitate the growth and survival of human embryonic stem cells by inhibiting anoikis. ROCK inhibitors are now part of standard stem cell culture protocols [51, 52]. Our results show that Y27632 can also increase the in vitro viability of MDA-MB-231 spheroids (Supplementary Figure 8). Therefore, it is possible Y27632 increased lung metastasis in MDA-MB-231 xenografts by promoting the survival of circulating tumor cells. We cannot either exclude that ROCK inhibition drove cancer cells toward a mesenchymal mode of migration [53]. In primary tumors, we found that Y27632 increased the recruitment of CAFs, PAI-1 expression and the phosphorylation of the proinvasive proteins ERM (Ezrin, Radixin, Moesin), even if ROCK is a known ERM activator [54, 55]. In contrast, PQ401 did not affect the phosphorylation of ERM proteins and the number of $\alpha$-SMA-positive cells. Although the blockade of ROCK reduces bone metastasis in MCF7 xenografts overexpressing ROCK [26], our findings suggest that in primary MDA-MB-231 tumors Y27632 can also promote CAF recruitment and stimulate proinvasive pathways, which may explain the increased lung metastasis formation.

In summary, we found that the crosstalk between CAFs and MDA-MB-231 BC cells increases the expression of IGF-1 in CAFs and PAI-1 activity in cancer cells. The increased expression of both IGF-1 and PAI-1 enhances RhoA signaling in cancer cells, which promotes cell scattering and invasion.

\section{MATERIALS AND METHODS}

\section{Cell culture}

Human mesenchymal-like breast cancer cell lines MDA-MB-231 (231) and MDA-MB-436 (436) were 
obtained from the American Type Culture Collection (ATCC). Cells were cultured in low glucose Dulbecco's modified Eagle's medium (DMEM) supplemented with $10 \% \mathrm{FBS}$ in a humidified 5\% CO2 incubator. Human breast CAF2 was obtained from Robert Weinberg [31]. The CAFs T53, T73 and T68 isolated from lesions of triple-negative breast cancer patients were characterized with antibodies as vimentin-positive and pan-cytokeratin-negative [32]. The Ast CAF (specimen 87322A1) - also isolated from triple-negative breast cancer lesions - were obtained from Asterand Inc. Normal human skin fibroblasts CRL-2575 were obtained from ATCC. All fibroblasts were cultured in DMEM supplemented with $10 \%$ FCS in a humidified $5 \% \mathrm{CO} 2$ incubator. HUVECs were acquired from the Center for Excellence in Vascular Biology, Brigham \& Women's Hospital, Harvard Medical School, Boston, MA and maintained in EGM medium (2\% FBS, brain bovine extract, heparin, hEGF, and hydrocortisone) (Lonza). Cancer cell spheroids were generated by seeding $2 \times 10^{5}$ cells/ml on low-adhesion surface (Corning) for $72 \mathrm{~h}$. For RNA and protein extraction, indirect co-culture of cancer cell spheroids and CAFs was performed in $0.4 \mu \mathrm{m}$ Transwell inserts (Corning).

\section{Antibodies and reagents}

Y-27632 (Tocris), geodin (Santa-Cruz), blocking human IGF-1 antibody (R\&D Systems, AF-291-NA) and recombinant IGF-1 (Sigma-Aldrich, I1146) were used in collagen invasion assay. The following antibodies: E-cadherin (Cell Signaling, \#3195), phospho-p120 catenin (Tyr228) (Cell Signaling, \#2911), PAI-1 (Cell Signaling, \#11907), phospho-ERM (Cell Signaling, \#3141), MLC (Cell Signaling, \#3672), phospho-MLC (Cell Signaling, \#3674), $\alpha$-SMA (Abcam, ab5694), $\alpha$-tubulin (Sigma-Aldrich, T5168), $\beta$-actin (Sigma-Aldrich, A5441) were used for immunoblotting or immunofluorescence on frozen sections. For in vivo experiments, Y27632 and PQ401 were obtained from AbMole Biosciences.

\section{Cell transfection}

Genetic silencing of RhoA in MDA-MB-231 and CAF2 was performed by transducting cells with a lentivirus-vector-based shRNA (Sigma-Aldrich, clone number TRCN0000047711). A non-target shRNA sequence was used as a control vector (Sigma-Aldrich, SHC002). Enhanced green fluorescent protein (EGF)expressing MDA-MB-231 cells were generated by transducing the cells with a pBMN-1-EGFP retroviral vector [56].

\section{In vitro collagen invasion assay}

$\mathrm{GFP}^{+}$expressing cancer cell spheroids $\left(10^{5}\right.$ cells $\left./ \mathrm{ml}\right)$ alone or in presence of CAFs $\left(5 \times 10^{4}\right.$ cells $\left./ \mathrm{ml}\right)$ were seeded in a $3 \mathrm{mg} / \mathrm{ml}$ collagen I-DMEM gels ( $\mathrm{pH}$ 7.2) and supplemented with $10 \%$ FCS. At this concentration, CAFs did not induce collagen gel contraction. In each experiment approximately 20 spheroids per condition were imaged with an inverted brightfield/epifluorescent microscope (Olympus IX70, PRIOR automated stage, OpenLab software) and $4 \times 1.00$ lens at multiple time points for seven days. Analysis of invasion areas and cell scattering were performed with ImageJ software. Briefly, invasion area was measured as an ellipse formed by the most distal $\mathrm{GFP}^{+}$cells from the spheroid center. The normalized invasion area was defined as the GFP ratio of the invasion area at a given time to size of the spheroid at d0. Cell scattering was defined as the number of GFPpositive single cells normalized to size of the spheroid at d1. The number of GFP-positive single cells was counted in automated fashion, after applying size- and intensitythresholds to eliminate non-specific signal.

\section{Gene expression analysis}

RNA was extracted using a RNeasy Mini Kit (Qiagen) and converted to cDNA using an iScript cDNA Synthesis Kit (Bio-Rad). The cDNA quality and concentration were measured with a ND-200 spectrophotometer (Nanodrop Technologies). Expression of EMT and motility-related genes was first determined by PCR array (Qiagen) and validated by RT-qPCR. RT-qPCR reaction was performed with Power SYBR Green Mix (Applied Biosystems). For human IGF1 and SERPINE1 detection, we used the following: IGF1 forward primer, 5'-AAGGAGGCTGGAGATGTATTGC-3'; IGF1 reverse primer, 5'-CGGACAGAGCGAGCTGACTT-3'; SERPINE1 forward primer, 5'-CACAAATCAGACGGCA GCACT-3'; SERPINE1 reverse primer, 5'-CATCGGGCG TGGTGAACTC'-3'. The relative gene expression was analyzed with the $2^{-\Delta \Delta \mathrm{Ct}}$ method.

\section{Immunoblotting}

Total proteins were extracted using RIPA buffer supplemented with protease and phosphatase inhibitor mixtures (Roche). Denatured proteins were analyzed on a reducing SDS-polyacrylamide gel and blotted to a PVDF membrane by electrotransfer. Membranes were blocked for $1 \mathrm{~h}$ at room temperature, with $5 \%$ non-fat dry milk in TBST and incubated overnight at $4{ }^{\circ} \mathrm{C}$ with primary antibodies. Protein detection was performed by using chemiluminescence with ECL or ECL+ reagents (GE healthcare). The relative intensity, shown as fold change over the control, was quantified with Image $\mathrm{J}$ software and represents the intensity of each protein band analyzed normalized to the intensity of either $\beta$-actin or GAPDH.

\section{RhoA activation assay}

To determine RhoA activation in cancer cells cocultured with CAFs, active RhoA-GTP was precipitated 
with Rhotekin-Rho-binding domain (RBD) glutathione affinity beads, using a RhoA Activation Assay Kit (Cytoskeleton) according to the manufacturer instructions. In tumor samples, RhoA activation was measured in tumor lysates with G-LISA RhoA Activation Assay Biochem Kit (Cytoskeleton).

\section{Detection of secreted IGF-1}

CAF were cultured alone or in presence of cancer cells in serum-free medium. Supernatants were collected after $72 \mathrm{~h}$ and IGF-1 was measured by ELISA, using human IGF-1 Quantikine ELISA kit (R\&D Systems) according to the manufacturer instructions. All IGF-1 values were normalized to amount of total proteins in the supernatant.

\section{In vivo tumor xenograft study}

$2 \times 10^{6}$ MDA-MB-231 cells were implanted in the mammary fat pad of SCID mice ( $\geq 13$ mice per group). Once tumors reached a tumor volume of approximately $40 \mathrm{~mm}^{3}$, mice were treated intraperitoneally with Y27632 [25 mg/kg], PQ401 [50 mg/kg] or Y27632 combined with PQ401, 3 times per week. Tumor volumes were measured every 2 to 3 days and volumes were based on the formula: $\frac{4}{3} \times \pi \times \frac{L}{2} \times\left(\frac{l}{2}\right)^{2}$. After 18 days, primary tumors were resected and processed for protein extraction or immunohistochemistry. Five weeks after primary tumor resection, mice were sacrificed and lung metastases were counted in whole lung sections stained with hematoxylin and eosin. Stained slides were quantified with Image J. The lung metastasis burden was defined as the fraction of image occupied by the metastases.

\section{Immunohistochemistry}

Resected tumors were fixed in $4 \%$ paraformaldehyde, soaked in sucrose solution for $24 \mathrm{~h}$ and embedded in optimum cutting temperature compound (OCT) (Sakura Finetek). Frozen sections were cut into $20 \mu \mathrm{m}$ sections, stained for $\alpha$-SMA and phospho-ERM and mounted in Vectashield with DAPI counterstain (Vector Laboratories). Confocal fluorescence images were acquired with an Olympus BX61WI microscope and $20 \times$ water immersion lens (Fluoview software). The content of each protein analyzed was determined by measuring the number of pixels above a threshold value that was set based on the average intensity value of pixels from all slides under analysis.

\section{Statistical analysis}

Data are expressed as mean \pm SEM. Statistical analyses were carried out using Prism 6.0 Software. The statistical significance between groups in normally distributed continuous variables was determined using Student's $t$-test, one-way or two way ANOVA coupled with Bonferroni's or Dunnett's post hoc test. For non-Gaussian distributed variables, the statistical significance between groups was determined using Mann-Whitney test or Kruskal-Wallis test coupled with Dunn's post hoc test. Tests were considered significant when $P$-values were $\leq 0.05$.

\section{ACKNOWLEDGMENTS}

The authors thank Drs João Incio, Ana Batista and Rosa Ng for their scientific input, and Dr Ned Kirkpatrick, Mark Duquette, Dannie Wang, Shan Min Chin, Anna Khachatryan, Dr Jieqiong Liu, Dr Peigen Huang, Sylvie Roberge, Carolyn Smith and Julia Kahn for their technical assistance.

\section{CONFLICTS OF INTEREST}

YB consultant Xtuit Inc.

\section{FUNDING}

The National Institute Grant R01-CA098706 supported this research (YB). The authors also thank the Fonds Quebecois de Recherche en Sante (FQRS), Réseau de Recherche sur le Cancer, Axe Cancer du Sein et Ovaire, Quebec Breast Cancer Foundation for biobank funding to provide the primary CAFs.

\section{REFERENCES}

1. Moorman AM, Vink R, Heijmans HJ, van der Palen J, Kouwenhoven EA. The prognostic value of tumour-stroma ratio in triple-negative breast cancer. Eur J Surg Oncol. 2012; 38:307-313.

2. Anton K, Glod J. Targeting the tumor stroma in cancer therapy. Curr Pharm Biotechnol. 2009; 10:185-191.

3. Polanska UM, Orimo A. Carcinoma-associated fibroblasts: non-neoplastic tumour-promoting mesenchymal cells. J Cell Physiol. 2013; 228:1651-1657.

4. McDonald LT, LaRue AC. Hematopoietic stem cell derived carcinoma-associated fibroblasts: a novel origin. Int J Clin Exp Pathol. 2012; 5:863-873.

5. Lecomte J, Masset A, Blacher S, Maertens L, Gothot A, Delgaudine $\mathrm{M}$, Bruyère $\mathrm{F}$, Carnet $\mathrm{O}$, Paupert J, Illemann $\mathrm{M}$, Foidart JM, Lund IK, Høyer-Hansen G, Noel A. Bone marrow-derived myofibroblasts are the providers of proinvasive matrix metalloproteinase 13 in primary tumor. Neoplasia. 2012; 14:943-951.

6. Hawsawi NM, Ghebeh H, Hendrayani SF, Tulbah A, Al-Eid M, Al-Tweigeri T, Ajarim D, Alaiya A, Dermime S, Aboussekhra A. Breast carcinoma-associated fibroblasts and their counterparts display neoplastic-specific changes. Cancer Res. 2008; 68:2717-2725. 
7. Polyak K, Haviv I, Campbell IG. Co-evolution of tumor cells and their microenvironment. Trends Genet. 2009; 25:30-38.

8. Catteau X, Simon P, Noel JC. Myofibroblastic stromal reaction and lymph node status in invasive breast carcinoma: possible role of the TGF-beta1/TGF-betaR1 pathway. BMC Cancer. 2014; 14:499.

9. Rajaram M, Li J, Egeblad M, Powers RS. System-wide analysis reveals a complex network of tumor-fibroblast interactions involved in tumorigenicity. PLoS Genet. 2013; 9:e1003789.

10. Shimoda M, Mellody KT, Orimo A. Carcinoma-associated fibroblasts are a rate-limiting determinant for tumour progression. Semin Cell Dev Biol. 2010; 21:19-25.

11. Goetz JG, Minguet S, Navarro-Lerida I, Lazcano JJ, Samaniego R, Calvo E, Tello M, Osteso-Ibanez T, Pellinen T, Echarri A, Cerezo A, Klein-Szanto AJ, Garcia R, et al. Biomechanical remodeling of the microenvironment by stromal caveolin-1 favors tumor invasion and metastasis. Cell. 2011; 146:148-163.

12. Taddei ML, Giannoni E, Comito G, Chiarugi P. Microenvironment and tumor cell plasticity: an easy way out. Cancer Lett. 2013; 341:80-96.

13. Yilmaz M, Christofori G. Mechanisms of motility in metastasizing cells. Mol Cancer Res. 2010; 8:629-642.

14. Kashiwagi S, Yashiro M, Takashima T, Nomura S, Noda S, Kawajiri H, Ishikawa T, Wakasa K, Hirakawa K. Significance of E-cadherin expression in triple-negative breast cancer. Br J Cancer. 2010; 103:249-255.

15. Andl CD, Rustgi AK. No one-way street: cross-talk between e-cadherin and receptor tyrosine kinase (RTK) signaling: a mechanism to regulate RTK activity. Cancer Biol Ther. 2005; 4:28-31.

16. Angelucci C, Maulucci G, Lama G, Proietti G, Colabianchi A, Papi M, Maiorana A, De Spirito M, Micera A, Balzamino OB, Di Leone A, Masetti R, Sica G. Epithelialstromal interactions in human breast cancer: effects on adhesion, plasma membrane fluidity and migration speed and directness. PLoS One. 2012; 7:e50804.

17. Yu Y, Xiao $\mathrm{CH}$, Tan LD, Wang QS, Li XQ, Feng YM. Cancer-associated fibroblasts induce epithelialmesenchymal transition of breast cancer cells through paracrine TGF-beta signalling. Br J Cancer. 2014; 110:724-732.

18. Evers EE, Zondag GC, Malliri A, Price LS, ten Klooster JP, van der Kammen RA, Collard JG. Rho family proteins in cell adhesion and cell migration. Eur J Cancer. 2000; 36:1269-1274.

19. Pankova K, Rosel D, Novotny M, Brabek J. The molecular mechanisms of transition between mesenchymal and amoeboid invasiveness in tumor cells. Cell Mol Life Sci. 2010; 67:63-71.

20. Sahai E, Marshall CJ. RHO-GTPases and cancer. Nat Rev Cancer. 2002; 2:133-142.
21. Taddei ML, Giannoni E, Morandi A, Ippolito L, Ramazzotti M, Callari M, Gandellini P, Chiarugi P. Mesenchymal to amoeboid transition is associated with stem-like features of melanoma cells. Cell Commun Signal. 2014; 12:24.

22. Demou ZN, Awad M, McKee T, Perentes JY, Wang X, Munn LL, Jain RK, Boucher Y. Lack of telopeptides in fibrillar collagen I promotes the invasion of a metastatic breast tumor cell line. Cancer Res. 2005; 65:5674-5682.

23. McHenry PR, Vargo-Gogola T. Pleiotropic functions of Rho GTPase signaling: a Trojan horse or Achilles' heel for breast cancer treatment? Curr Drug Targets. 2010; 11:1043-1058.

24. Ma L, Liu YP, Geng CZ, Wang XL, Wang YJ, Zhang XH. Over expression of RhoA is associated with progression in invasive breast duct carcinoma. Breast J. 2010; 16:105-107.

25. Katz E, Sims AH, Sproul D, Caldwell H, Dixon MJ, Meehan RR, Harrison DJ. Targeting of Rac GTPases blocks the spread of intact human breast cancer. Oncotarget. 2012; 3:608-19. https://doi.org/10.18632/oncotarget.520.

26. Liu S, Goldstein RH, Scepansky EM, Rosenblatt M. Inhibition of rho-associated kinase signaling prevents breast cancer metastasis to human bone. Cancer Res. 2009; 69:8742-8751.

27. Wolf K, Wu YI, Liu Y, Geiger J, Tam E, Overall C, Stack MS, Friedl P. Multi-step pericellular proteolysis controls the transition from individual to collective cancer cell invasion. Nat Cell Biol. 2007; 9:893-904.

28. Liu YJ, Le Berre M, Lautenschlaeger F, Maiuri P, Callan-Jones A, Heuze M, Takaki T, Voituriez R, Piel M. Confinement and low adhesion induce fast amoeboid migration of slow mesenchymal cells. Cell. 2015; 160:659-672.

29. Sanz-Moreno V, Gaggioli C, Yeo M, Albrengues J, Wallberg F, Viros A, Hooper S, Mitter R, Feral CC, Cook M, Larkin J, Marais R, Meneguzzi G, et al. ROCK and JAK1 signaling cooperate to control actomyosin contractility in tumor cells and stroma. Cancer Cell. 2011; 20:229-245.

30. Gaggioli C, Hooper S, Hidalgo-Carcedo C, Grosse R, Marshall JF, Harrington K, Sahai E. Fibroblast-led collective invasion of carcinoma cells with differing roles for RhoGTPases in leading and following cells. Nat Cell Biol. 2007; 9:1392-1400.

31. Orimo A, Gupta PB, Sgroi DC, Arenzana-Seisdedos F, Delaunay T, Naeem R, Carey VJ, Richardson AL, Weinberg RA. Stromal fibroblasts present in invasive human breast carcinomas promote tumor growth and angiogenesis through elevated SDF-1/CXCL12 secretion. Cell. 2005; 121:335-348.

32. Hosein AN, Wu M, Arcand SL, Lavallee S, Hebert J, Tonin PN, Basik M. Breast carcinoma-associated fibroblasts rarely contain p53 mutations or chromosomal aberrations. Cancer Res. 2010; 70:5770-5777.

33. Castano J, Solanas G, Casagolda D, Raurell I, Villagrasa P, Bustelo XR, Garcia de Herreros A, Dunach M. Specific phosphorylation of p120-catenin regulatory domain 
differently modulates its binding to RhoA. Mol Cell Biol. 2007; 27:1745-1757.

34. Gable KL, Maddux BA, Penaranda C, Zavodovskaya M, Campbell MJ, Lobo M, Robinson L, Schow S, Kerner JA, Goldfine ID, Youngren JF. Diarylureas are small-molecule inhibitors of insulin-like growth factor I receptor signaling and breast cancer cell growth. Mol Cancer Ther. 2006; 5:1079-1086.

35. Zielinska HA, Bahl A, Holly JM, Perks CM. Epithelial-tomesenchymal transition in breast cancer: a role for insulinlike growth factor I and insulin-like growth factor-binding protein 3? Breast Cancer (Dove Med Press). 2015; 7:9-19.

36. Zhang XH, Jin X, Malladi S, Zou Y, Wen YH, Brogi E, Smid M, Foekens JA, Massague J. Selection of bone metastasis seeds by mesenchymal signals in the primary tumor stroma. Cell. 2013; 154:1060-1073.

37. Dunn SE, Ehrlich M, Sharp NJ, Reiss K, Solomon G, Hawkins R, Baserga R, Barrett JC. A dominant negative mutant of the insulin-like growth factor-I receptor inhibits the adhesion, invasion, and metastasis of breast cancer. Cancer Res. 1998; 58:3353-3361.

38. Weigel KJ, Jakimenko A, Conti BA, Chapman SE, Kaliney WJ, Leevy WM, Champion MM, Schafer ZT. CAF-secreted IGFBPs regulate breast cancer cell anoikis. Mol Cancer Res. 2014; 12:855-866.

39. Peruzzi F, Prisco M, Dews M, Salomoni P, Grassilli E, Romano G, Calabretta B, Baserga R. Multiple signaling pathways of the insulin-like growth factor 1 receptor in protection from apoptosis. Mol Cell Biol. 1999; 19:7203-7215.

40. Fang H, Placencio VR, DeClerck YA. Protumorigenic activity of plasminogen activator inhibitor-1 through an antiapoptotic function. J Natl Cancer Inst. 2012; 104:1470-1484.

41. Marudamuthu AS, Bhandary YP, Shetty SK, Fu J, Sathish V, Prakash Y, Shetty S. Role of the urokinase-fibrinolytic system in epithelial-mesenchymal transition during lung injury. Am J Pathol. 2015; 185:55-68.

42. Chazaud B, Ricoux R, Christov C, Plonquet A, Gherardi RK, Barlovatz-Meimon G. Promigratory effect of plasminogen activator inhibitor-1 on invasive breast cancer cell populations. Am J Pathol. 2002; 160:237-246.

43. Cartier-Michaud A, Malo M, Charriere-Bertrand C, Gadea G, Anguille C, Supiramaniam A, Lesne A, Delaplace F, Hutzler G, Roux P, Lawrence DA, Barlovatz-Meimon G. Matrix-bound PAI-1 supports cell blebbing via RhoA/ ROCK1 signaling. PLoS One. 2012; 7:e32204.

44. Masuda T, Hattori N, Senoo T, Akita S, Ishikawa N, Fujitaka K, Haruta Y, Murai H, Kohno N. SK-216, an inhibitor of plasminogen activator inhibitor-1, limits tumor progression and angiogenesis. Mol Cancer Ther. 2013; 12:2378-2388.

45. Konrad L, Scheiber JA, Schwarz L, Schrader AJ, Hofmann R. TGF-beta1 and TGF-beta2 strongly enhance the secretion of plasminogen activator inhibitor-1 and matrix metalloproteinase- 9 of the human prostate cancer cell line PC-3. Regul Pept. 2009; 155:28-32.

46. Pan XY, Wang Y, Su J, Huang GX, Cao DM, Qu S, Lu J. The mechanism and significance of synergistic induction of the expression of plasminogen activator inhibitor-1 by glucocorticoid and transforming growth factor beta in human ovarian cancer cells. Mol Cell Endocrinol. 2015; 407:37-45.

47. Kruithof EK. Regulation of plasminogen activator inhibitor type 1 gene expression by inflammatory mediators and statins. Thromb Haemost. 2008; 100:969-975.

48. Lane J, Martin TA, Watkins G, Mansel RE, Jiang WG. The expression and prognostic value of ROCK I and ROCK II and their role in human breast cancer. Int J Oncol. 2008; 33:585-593.

49. Pille JY, Denoyelle C, Varet J, Bertrand JR, Soria J, Opolon P, Lu H, Pritchard LL, Vannier JP, Malvy C, Soria C, Li H. Anti-RhoA and anti-RhoC siRNAs inhibit the proliferation and invasiveness of MDA-MB-231 breast cancer cells in vitro and in vivo. Mol Ther. 2005; 11:267-274.

50. Narumiya S, Tanji M, Ishizaki T. Rho signaling, ROCK and mDia1, in transformation, metastasis and invasion. Cancer Metastasis Rev. 2009; 28:65-76.

51. Motomura K, Okada N, Morita H, Hara M, Tamari M, Orimo K, Matsuda G, Imadome KI, Matsuda A, Nagamatsu T, Fujieda M, Sago H, Saito H, et al. A Rhoassociated coiled-coil containing kinases (ROCK) inhibitor, Y-27632, enhances adhesion, viability and differentiation of human term placenta-derived trophoblasts in vitro. PLoS One. 2017; 12:e0177994.

52. Wen J, Zu T, Zhou Q, Leng X, Wu X. Y-27632 simplifies the isolation procedure of human primary epidermal cells by selectively blocking focal adhesion of dermal cells. J Tissue Eng Regen Med. 2017.

53. Jiang P, Enomoto A, Takahashi M. Cell biology of the movement of breast cancer cells: intracellular signalling and the actin cytoskeleton. Cancer Lett. 2009; 284:122-130.

54. Croft DR, Sahai E, Mavria G, Li S, Tsai J, Lee WM, Marshall CJ, Olson MF. Conditional ROCK activation in vivo induces tumor cell dissemination and angiogenesis. Cancer Res. 2004; 64:8994-9001.

55. Hebert M, Potin S, Sebbagh M, Bertoglio J, Breard J, Hamelin J. Rho-ROCK-dependent ezrin-radixin-moesin phosphorylation regulates Fas-mediated apoptosis in Jurkat cells. J Immunol. 2008; 181:5963-5973.

56. Samuel R, Daheron L, Liao S, Vardam T, Kamoun WS, Batista A, Buecker C, Schafer R, Han X, Au P, Scadden DT, Duda DG, Fukumura D, et al. Generation of functionally competent and durable engineered blood vessels from human induced pluripotent stem cells. Proc Natl Acad Sci USA. 2013; 110:12774-12779. 